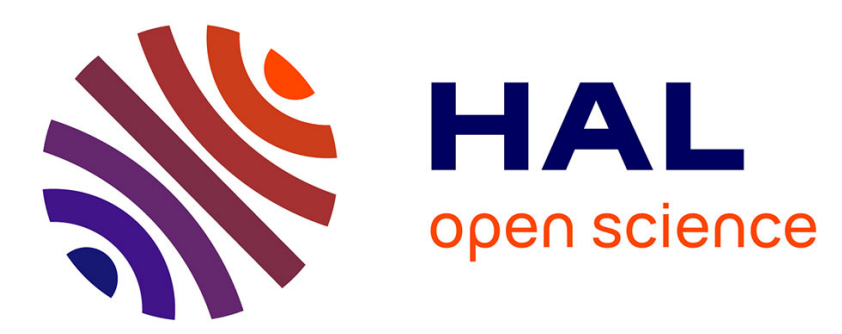

\title{
Observer-Based Efficiency Enhancement in Cell-Cycle Specific Therapies
}

Mazen Alamir, Mirko Fiacchini

\section{To cite this version:}

Mazen Alamir, Mirko Fiacchini. Observer-Based Efficiency Enhancement in Cell-Cycle Specific Therapies. CDC 2016 - 55th IEEE Conference on Decision and Control, Dec 2016, Las Vegas, NV, United States. hal-01348893

\section{HAL Id: hal-01348893 https://hal.science/hal-01348893}

Submitted on 29 Jul 2016

HAL is a multi-disciplinary open access archive for the deposit and dissemination of scientific research documents, whether they are published or not. The documents may come from teaching and research institutions in France or abroad, or from public or private research centers.
L'archive ouverte pluridisciplinaire HAL, est destinée au dépôt et à la diffusion de documents scientifiques de niveau recherche, publiés ou non, émanant des établissements d'enseignement et de recherche français ou étrangers, des laboratoires publics ou privés. 


\title{
Observer-Based Efficiency Enhancement in Cell-Cycle Specific Therapies
}

\author{
Mazen Alamir and Mirko Fiacchini
}

\begin{abstract}
Cell-cycle specific drugs affect cells when they are in specific phases of their periodic cycle. However, injecting drug's strategy can only by global. This rises the problem of optimizing a global constrained decision variable (drug delivery profile with limited volume) when its effect depends on many local unknown characteristics (the individual cells phases). In this paper, an observer-based framework is proposed that enables a rational decision making in this particular circumstance. Simulation on a simple case are proposed to show the relevance of the proposed framework.
\end{abstract}

\section{INTRODUCTION}

The cell cycle is the sequence of phases leading to the cell division. It is composed of four main phases during which the cell prepares for the DNA synthesis (G1); duplicates the DNA (S); and finally, after a phase (G2) of preparation, reaches the mitosis phase $(\mathrm{M})$ in which the cell is divided, see [16]. Checkpoints are present along the cycle to ensure that the cell is ready for proceeding in the division process [15]. Such a complex process is driven by some proteins (CDK) and regulated through the apoptosis, that is a programmed cell death. The tumoral cells are characterized by abnormal growth as well as mutations of genes, like the p53 responsible for the cell cycle regulation and apoptosis, resulting in an uncontrolled cells proliferation.

In parallel with standard chemotherapeutic agents, highly toxic and non-selective in their targets, novel compounds have been developed in the last decades that act on the cell cycle-regulating molecules to interfere in the cell divisions and to induce the apoptosis in the tumoral cells, [4], [7], [10], [13]. These new cell cycle-specific inhibitors, together with a deeper knowledge of the cell cycle, represent a possible solution to overcome the tumoral resistance to cytotoxic drugs. The appropriate sequencing and scheduling of several cell cycle-specific agents can reduce the tumor drug resistance and induce biochemical synergy (i.e. an efficiency greater than the additive ones), by targeting different processes in the cell cycle, see [12]. The evolution of the cell cycle-mediated drug resistance, or kinetics resistance, has been addressed by means of mathematical and computational models, summarized in [5], employed also for control-based therapeutic design, see [14], [9].

While some therapy agents are able to kill a cell during all the phases of its cycle, cell-cycle specific agents are efficient

This work was not supported by any organization

M. Alamir and M. Fiacchini are with CNRS, Gipsa-lab, Control System Department, University of Grenoble Alpes. 11, rue des Mathématiques, Grenoble, France. only when the cell is in a (set) of specific phases of its cycle [5]. Moreover, the distribution of phases over a given population of tumor cells is unlikely to be uniform, that is to say, the phase-in-the-cycle is an individual component of each cell's current state description. On the other hand, the drug delivery is global whether it is taken orally or intravenously and can therefore not be administered cell-bycell should the individual phases be known. Even when the so-called direct advanced delivery methods are concerned [11], the local areas being involved still englobe too many cells with heterogeneous phase distribution. This raises the obvious question regarding the way the time delivery of a limited quantity of drug has to be administered so that its efficiency is enhanced despite the lack of measurement of the phase distribution over the population for a given prediction horizon.

In this contribution, a tentative framework is proposed to address this difficult problem. The framework is based on a simple model and an associated observer design together with an associated drug delivery strategy that is based on the so reconstructed unknown aggregated quantity.

As far as the representation of the cell-cycle effect on the drug's efficiency is concerned, a phase-dependent gain affecting the drug's effect term is used in which a periodic phase description is considered with cell-dependent unknown internal state. Regarding the dynamics, a standard population dynamics is considered in which the effect of drug is represented as a sum over all individual cells involved in the population description.

The case of a single chemotherapy drug is used for illustration purposes in order to focus on the main contribution. The latter can obviously be generalized to any combined therapy in which at least one of the drugs is cell-cycle-specific.

The paper is organized as follows: The mathematical model used in the study is first introduced in section II, the control problem being addressed is clearly stated in Section III. In section IV, the observer-based proposed solution to the problem of enhancing the drug efficiency under partial knowledge and limited amount of available drug is derived. Section V gives some simulations that compare the efficiency of the proposed strategy to an intuitive alternatives in order to assess the benefit from the use of the observer-based drug administration profile. 
Finally section VI concludes the paper and gives hints for further investigation.

\section{MATHEMATICAL MODEL}

Consider the dynamic model given by:

$$
\begin{aligned}
\dot{T} & =f(T)-T\left[\sum_{j=1}^{m} \sum_{i=1}^{q} \frac{\kappa_{j}}{q}\left(1-\cos \left(\varphi_{i j}\right)\right)\right] D \\
\dot{\varphi}_{i j} & =\omega_{j}+\delta_{i j} \\
\dot{D} & =-\lambda D+\alpha u
\end{aligned}
$$

where $T$ is the size of the tumor cells population, $D$ is the concentration of drug, $u$ is the drug delivery intensity (the control input), $q$ is the number of individual cells in the population, $m$ is the number of modes with constant pulsations

$$
\omega_{j}:=2 j \pi / T_{c} \quad, \quad j=1, \ldots, m
$$

in which $T_{c}$ stands for the period of the total sequence of cycles, $\left\{\delta_{i j}\right\}_{i=1}^{q}$ describe the discrepancy of the $j$-th mode pulsation value at the $i$-th cell's level. Regarding this discrepancies, it is assumed that:

$$
\left|\delta_{i j}\right| \ll \omega_{j}
$$

The term $f(T)$ in (1) describes the drug-free evolution of the tumor that can takes many forms in the literature (see for instance [8], [3], [6] and the references therein). The precise definition of this term is not necessary at this stage. Finally $\lambda$ and $\left\{\kappa_{j}\right\}_{j=1}^{m}$ are known positive constants describing the drug dissolution rate and the periodic form of the cell-cycle specific impact of the chemotherapy during the cell cycle. More precisely, the following time profile's is supposed to faithfully describe the gain with which the drug concentration $D$ affects an individual tumor cell as a function of the time $t$ inside the cell's cycle in a discrepancy-free case $\left(\delta_{i j}=0\right)$ :

$$
\frac{1}{q} \sum_{j=1}^{m} \kappa_{j}\left[1-\cos \left(\omega_{j} t+\theta_{j}\right)\right]
$$

which is obviously sufficiently general to represent a wide variety of cell-cycle specific drug's effect variations.

Note that (6) leads to the second term in the r.h.s of (1) by taking the sum over all individual cells, introducing the pulsation discrepancies $\delta_{i j}$ and defining $\varphi_{i j}$ to be the argument of the cos function in (6).

Remark 1: Note that the basic period $T_{c}$ involved in the definition of the modes through (4) and the needed number $m$ of modes that are necessary to describe the cycle-specific behavior of the drug through the gain (6) represent a priori knowledge for the present contribution. This knowledge is drug-dependent and should be viewed as a drug's characterization which implicitly includes the knowledge of the coefficients $\kappa_{j}, j=1, \ldots, m$.
In the sequel, the following notation is used to simplify the expressions:

$$
G:=\sum_{j=1}^{m} G_{j} ; G_{j}:=\sum_{i=1}^{q} \frac{\kappa_{j}}{q} g_{i j} ; g_{i j}=1-\cos \left(\varphi_{i j}\right)
$$

so that equation (1) becomes:

$$
\dot{T}=f(T)-T G D
$$

\section{Problem Statement}

Cancer treatment protocols are generally defined by a sequence of treatment periods separated by rest periods during which no drug is delivered. Since this paper focuses on deriving a rational computation of appropriate drug injection profiles during the treatment periods, we consider only a sequence of several successive days of treatment. Moreover, we assume without loss of generality that the basic cycles period $T_{c}$ involved in the definition (4) of the pulsations $\omega_{j}$ invoked in the model [see equation (2)] is equal to unity (one day) which is a reasonable order of magnitude [2].

Let us use $k$ to index the successive periods of length $T_{c}$ denoted hereafter by $\mathcal{T}_{k}=\left[t_{k}, t_{k+1}\right]$ where $t_{k}=k T_{c}$. Denote by $u_{k}(\tau)$ the intensity of drug delivery at instant $t_{k}+\tau, \tau \in\left[0, T_{c}\right]$ inside the $k$-th period $\mathcal{T}_{k}$.

The control profile $u_{k}(\cdot)$ is constrained to be a piecewise constant function of time with a sampling period of $\tau_{s}$ $\left(2,3,4\right.$ or 6 hours are typical candidate values for $\left.\tau_{s}\right)$. In what follows it is assumed that $T_{c}$ is a multiple $N_{s}$ of the sampling period $\tau_{s}$, namely:

$$
T_{c}=N_{s} \tau_{s}
$$

Finally, it is assumed that some outer loop computation procedure taking into account the total amount of available drug for the whole treatment delivers a sequence of available amounts $\left\{\Delta_{k}\right\}_{k=1}^{M}$ of drugs for the $M$ treatment periods $\mathcal{T}_{k}$. More precisely, the following constraints have to be satisfied:

$$
\forall k \in\{1, \ldots, M\}, \quad \int_{0}^{T_{c}} u_{k}(\tau) d \tau \leq \Delta_{k}
$$

Note that the bounds $\Delta_{k}$, can be optimized by an outer loop that assigns these upper bounds over the coming intervals while using in the inner loop the optimization of the control profiles proposed in this contribution for a given set of upper bounds $\Delta_{k}$.

Regarding the updating period of the injection profiles, it is assumed that at the beginning of each interval $\mathcal{T}_{k}$, the corresponding injection profile $u_{k}(\cdot)$ is computed (based on the past measurements collected during the past periods $\mathcal{T}_{k^{\prime}}, k^{\prime}<k$ ) and applied in open-loop during $\mathcal{T}_{k}$, the data collected during $\mathcal{T}_{k}$ is then used (together with the past measurements) to compute the injection profile $u_{k+1}(\cdot)$ to be applied on $\mathcal{T}_{k+1}$ and so on. 
Based on the above discussion, the problem addressed in the current contribution can be stated as follows:

\section{Problem Statement:}

Use the measured quantities $D$ and $T$ to compute, at the beginning of each period $\mathcal{T}_{k}$, an optimal injection profile $\boldsymbol{u}_{k}^{*}:=u_{k}^{*}(\cdot)$ satisfying the constraints (10) while maximizing the drug's effect on the tumor size.

In the next section, an observer-based solution to the above stated problem is introduced.

\section{DERIVATION OF THE PROPOSED FEEDBACK}

The starting point of the proposed solution is to observe that if one can perfectly predict the evolution of $G$ over $\mathcal{T}_{k}$, denoted hereafter by $G_{k}(\tau):=G\left(t_{k}+\tau\right), \tau \in\left[0, T_{c}\right]$, then the optimal injection profile would be given by the solution of the constrained optimization problem:

$$
\begin{aligned}
\mathcal{P}_{k}\left(G_{k}(\cdot)\right): & \boldsymbol{u}_{k}^{*} \leftarrow \max _{u_{k}(\cdot)}\left[\int_{0}^{T_{c}} D_{k}(\tau) G_{k}(\tau) d \tau\right] \\
& \text { under the constraints }(\forall \tau) \\
& \dot{D}_{k}(\tau)=-\lambda D_{k}(\tau)+\alpha u_{k}(\tau) \\
& \int_{0}^{T_{c}} u_{k}(\tau) d \tau \leq \Delta_{k} \\
& 0 \leq u_{k}(\tau) \leq u_{\text {max }}
\end{aligned}
$$

which is obviously an easy-to-solve Linear Progamming (LP) problem in the decision variable $\boldsymbol{u}_{k} \in \mathbb{R}_{+}^{N_{s}}$ representing the piece-wise constant nature of the control profile $u_{k}(\cdot)$ :

$$
\boldsymbol{u}_{k}:=\left(\begin{array}{c}
u_{k}(0) \\
u_{k}\left(\tau_{s}\right) \\
\vdots \\
u_{k}\left(T_{c}-\tau_{s}\right)
\end{array}\right)
$$

which are the successive constant values to be applied over the sampling periods contained in $\mathcal{T}_{k}$.

Note that the cost function (11) maximizes the drug's effect by accommodating for high values of $D$ over the sub-interval of $\mathcal{T}_{k}$ where the gain $G_{k}$ is the most important. This is done by taking into account the dynamic of the drug concentration, the available upper bound on the drug delivery during the interval and on the necessarily positive nature of the control variable. Moreover, an upper bound $u_{\max }$ on the maximal intensity of the injection is also introduced in the r.h.s of (14).

It comes from the preceding discussion that the prediction of the future evolution $G_{k}(\cdot)$ over the interval $\mathcal{T}_{k}$ that is used in the expression of the cost function (11) is the key task in achieving the control objective. Indeed, once such a prediction $\hat{G}_{k}(\cdot)$ is available, the optimal injection profile can be obtained by solving the associated LP problem $\mathcal{P}_{k}\left(\hat{G}_{k}(\cdot)\right)$.
In the remainder of this section, it is shown that such a prediction $\hat{G}_{k}(\cdot)$ can be achieved by mean of dynamic state estimation. To do so, the following Lemma is needed:

Lemma 1: The evolution of the gain $G$ involved in (8) obeys the following dynamic equations:

$$
\begin{aligned}
\ddot{z}_{j} & =-\omega_{j}^{2} z_{j}-\epsilon_{j} \quad ; \quad j=1, \ldots, m \\
G & =\sum_{j=1}^{m}\left(z_{j}+\kappa_{j}\right)
\end{aligned}
$$

where $\left|\epsilon_{j}\right| \ll\left|\omega_{j}^{2} z_{j}\right|$.

Proof. Using the definition $g_{i j}=1-\cos \left(\varphi_{i j}\right)$, it comes that:

$$
\ddot{g}_{i j}=-\omega_{j}^{2}\left(g_{i j}-1\right)-2 \omega_{j} \delta_{i j}\left(g_{i j}-1\right)
$$

Using this to compute $G_{j}$ according to (7) gives:

$$
\ddot{G}_{j}=-\omega_{j}^{2} G_{j}+\kappa_{j} \omega_{j}^{2}-\epsilon_{j}
$$

where

$$
\epsilon_{j}:=\frac{2}{q} \sum_{i=1}^{q} \kappa_{j} \omega_{j} \delta_{i j}\left(g_{i j}-1\right)
$$

Using the following notation:

$$
\eta_{j}:=\max _{i \in\{1, \ldots, q\}}\left[\frac{\delta_{i j}}{\omega_{j}}\right] \ll 1
$$

the following bound can be derived on $\left|\epsilon_{i j}\right|$ :

$$
\begin{aligned}
\left|\epsilon_{i j}\right| & \leq \frac{2}{q} \eta_{j} \sum_{i=1}^{q} \kappa_{j} \omega_{j}^{2}\left(1-g_{i j}\right) \\
& =2 \eta_{j} \omega_{j}^{2}\left(G_{j}-\kappa_{j}\right) \ll \omega_{j}^{2}\left|G_{j}-\kappa_{j}\right|
\end{aligned}
$$

where the last inequality comes from (21). Now using the notation $z_{j}=G_{j}-\kappa_{j}$, the result follows.

The consequence of Lemma 1 is that one can approximately predict the future evolution of $G$ provided that the state of the dynamic system (16) can be observed. But since $\omega_{j}$ are different, the estimation of the state:

$$
z=\left(\begin{array}{lllll}
z_{1} & \dot{z}_{1} & \ldots & z_{m} & \dot{z}_{m}, e
\end{array}\right)^{T} \in \mathbb{R}^{2 m+1}
$$

can be done if a good estimation $\hat{G}$ (and not prediction) of $G$ can be delivered. Indeed, in this case, a standard (Luenberger or Kalman)-like observer can be built for the dynamic system:

$$
\dot{z}=A z \quad ; \quad y=C z
$$

where

$$
\begin{aligned}
& A:=\operatorname{diag}\left(\left\{\left(\begin{array}{cc}
0 & 1 \\
-\omega_{j}^{2} & 0
\end{array}\right)\right\}_{j=1}^{m}, 0\right) \\
& \left.C:=\left(\begin{array}{ll}
1 & 0
\end{array}\right) \otimes \mathbf{1}^{T} \quad 1\right)
\end{aligned}
$$

Indeed, (23) represents (16) in which $\epsilon_{j}=0$ is used while an additional scalar $e$ is added to accommodate for a drift in 
$G$ that might be due to the presence of small terms $\epsilon_{j}$. Note that by virtue of (17), the measurement $y$ is precisely given by:

$$
y=\hat{G}-\sum_{j=1}^{m} \kappa_{j}
$$

The fact that (23) is observable is obvious and is not explicitly proved here as it directly follows from a careful examination of the observability rank. Instead, the way a reconstruction of an estimation $\hat{G}$ of $G$ is explained hereafter since it is used in the delivery of the output $y$ in (23) according to (26).

In order to estimate $G$, the dynamic equation (8) of the tumor is rewritten by using the notation $\psi=-T G D$ :

$$
\dot{T}=\psi+f(T)
$$

Now since $T$ is measured and $f(T)$ is assumed to be known, a high gain observer can enable recovering $\psi$ by building a dynamic observer to the following dynamic system

$$
\dot{T}=\psi+f(T) \quad ; \quad \dot{\psi}=0
$$

This is possible because this system in the state $(T, \psi)$ and the exogenous measured signal $f(T)$ involves the state matrix $\left(\begin{array}{ll}0 & 1 \\ 0 & 0\end{array}\right)$ and the measurement matrix ( $\left.1 \begin{array}{ll}1 & 0\end{array}\right)$ which obviously corresponds to an observable pair with an observability matrix that is equal to the identity matrix in $\mathbb{R}^{2 \times 2}$. Based on the estimation $\hat{\psi}$ of the state component $\psi$, the estimation of $G$ can then be given by:

$$
\hat{G}=-\frac{\hat{\psi}}{T D}
$$

Finally, given the specific nature of the problem where continuous measurements are not available, reconstruction algorithms should be designed in a discret-time setting using a measurement sampling period $\tau_{m}$. In the statement of the following result, the notation $\bar{A}_{1}$ denotes the $\tau_{m}$-discrete version of the matrix $A$ defined by (24). Moreover $\bar{A}_{2}$ and $\bar{B}_{2}$ denote the $\tau_{m}$-discrete versions of the $(A, B)$ pair involved in the description of the two-dimensional system (28).

Proposition 1: There is two computable gain matrices $L_{1} \in \mathbb{R}^{2 m+1}$ and $L_{2} \in \mathbb{R}^{2}$ such that the following dynamic system is an approximate observer of $\hat{z}$ and $\hat{G}$ (the superscript ${ }^{+}$denotes the next measurement sampling period related quantities):

$$
\begin{aligned}
{\left[\begin{array}{c}
\hat{T} \\
\hat{\psi}
\end{array}\right]^{+} } & =\bar{A}_{2}\left[\begin{array}{l}
\hat{T} \\
\hat{\psi}
\end{array}\right]+\frac{\bar{B}_{2}}{2}\left[f(T)+f\left(T^{+}\right)\right]+L_{2}(T-\hat{T}) \\
\hat{z}^{+} & =\bar{A}_{1} \hat{z}+L_{1}\left[-\frac{\hat{\psi}}{T D}-\sum_{j=1}^{m} \kappa_{j}-C \hat{z}\right] \\
\hat{G} & =-\frac{\hat{\psi}}{T D}
\end{aligned}
$$

Moreover, the gains $L_{1}$ and $L_{2}$ are any discrete-time observer gains for the dynamic system (23) and (28) which are observable linear (up to an output injection) systems.

PROOF. Straightforward given the above discussion. Note simply that the second term in the r.h.s of the first equation approximates the average of $f(T)$ over the measurement period as the time discretization are based on a piece-wise constant exogenous signals.

Having the estimation of the state $\hat{z}\left(t_{k}\right)$ at instant $t_{k}$, the prediction $\hat{G}_{k}(\cdot)$ is given by:

$$
\hat{G}_{k}(\tau):=C \tilde{z}\left(t_{k}+\tau \mid \hat{z}\left(t_{k}\right)\right)+\sum_{j=1}^{m} \kappa_{j}
$$

where $\tilde{z}\left(t_{k}+\tau \mid \hat{z}\left(t_{k}\right)\right)$ is the prediction of future evolution of $z$ based on the dynamic model $\dot{z}=A z$ and the initial state $\hat{z}\left(t_{k}\right)$.

This estimation can then be used in the formulation of the $\mathbf{L P}$ optimization problem $\mathcal{P}\left(\hat{G}_{k}(\cdot)\right)$ defined by (11)-(14) leading to the injection profile $\boldsymbol{u}_{k}^{*}$ one is looking for. This solves the problem stated in Section III.

Remark 2: It is worth underlying that the number $q$ of cells used in the description of the dynamic model does not appear in the solutions's algorithms. Namely, $q$ is not explicitly involved neither in the observer's equations of Proposition 1 nor in the expression of the LP problem that is used to derive the optimal injection profile. This feature is induced by the fact that $\kappa_{j}$ is an aggregated quantity related to the set of cells and not to a single cell. That is why $q$ appears in the denominator of the gain expressed in (6). $\diamond$

\section{ILLUSTRATIVE EXAMPLES}

Let us consider the drug-free evolution term proposed in [6], [1]:

$$
f(T):=a T(1-b T)
$$

where $a=4.31 \times 10^{-3} \mathrm{day}^{-1}$ and $b=1.02 \times 10^{-14}$ cell $^{-1}$. Simulations are done in the case where $m=3$ modes describe the evolution of the drug gain term involved in (1) and (2). Two different possible values of $T_{c} \in\{1,1.1\}$ are simulated in the sequel. The coefficient $\kappa_{j}, j=1, \ldots, 3$ are given by:

$$
\kappa_{1}=3 \times 10^{-1} ; \kappa_{2}=6 \times 10^{-3} ; \kappa_{3}=3 \times 10^{-2}
$$

The coefficients $\lambda$ and $\alpha$ involved in equation (3) describing the evolution of the drug concentration is given by $\lambda=10$ and $\alpha=0.1$.

The maximal intensity of drug injection $u_{\max }$ used in the definition of the control computation through (14) is given by $u_{\max }=8$ while the maximum quantity of injected drug during a cycle [see equation (13)] is taken uniform (along the cycles) and two values are simulated $\Delta_{k} \in\{4,1\}$. 
The sampling period $\tau_{s}$ that defines the piece-wise constant control is given by $\tau_{s}=0.1$ day, namely 144 minutes (2.4 hour). The basic sampling period for the measurement is taken equal to $\tau_{m}=0.01$ day, namely 14.4 minutes.

The proposed strategy is compared to a drug injection strategy in which a maximal uniform drug injection is used, namely a strategy in which

$$
u_{k}\left(t_{k}+\tau\right):=u^{\dagger}=\frac{\Delta_{k}}{T_{c}}
$$

which obviously satisfies the constraints (13)-(14) while injecting the maximal quantity of drug over each cycle.

The unknown discrepancies $\delta_{i j}$ on the pulsation are introduced such that:

$$
\delta_{i 1}=0.04 ; \delta_{i 2}=-0.06 ; \delta_{i 3}=0.12
$$

Note that in the forthcoming simulations, the proposed strategy is fired only at the beginning of the second cycle in order to avoid the initial transient on the observer's states. That is the reason why in all the simulations, the behavior of the system under the two strategies coincide during the first cycle.

In the sequel, two scenarios are simulated in which the pair $\left(T_{c}, \Delta_{k}\right)$ takes respectively the values $(1,4)$ and $(1.1,1)$. Figures $1-4$ concern the first scenario while Figures 5 and 6 concern the second scenario. More precisely:

- Figure 1 shows the evolution of the tumor size for the first scenario $\left[\left(T_{c}, \Delta_{k}\right)=(1,4)\right]$. It clearly shows that the proposed observer-based injection strategy enhances the efficiency of the drug on towards the contraction of the tumor.

- Figure 2 shows the evolution of the phase-dependent gain $G$ and its observer-based estimation $\hat{G}$. One can note that the evolution is not purely periodic because of the discrepancies on the pulsations $\omega_{j}$ that are introduced through the parameters $\delta_{i j}$. The Figure shows however good estimation profile despite the absence of asymptotic convergence which is due to the approximation $\dot{\psi}=0$ which is obviously not rigorously satisfied.

- Figure 3 shows a zoom representing the evolution of $G$ and $\hat{G}$ during four successive cells cycles. This Figure suggests that for the simulated case, the gain varies by a factor 4 during a single cycle. This obviously suggests that a gain-dependent strategy is likely to perform better performance that a constant uniform strategy.

- Figure 4 shows the evolution of the drug injection intensity for the two competing strategies. More precisely, while the uniform maximal strategy corresponds to a

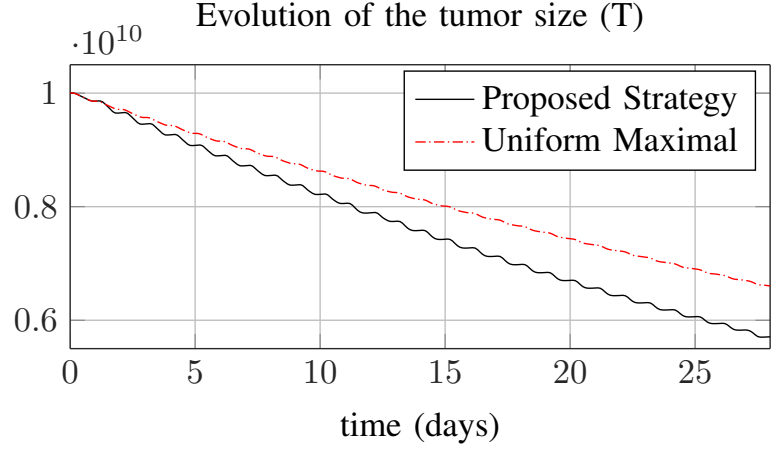

Fig. 1. Case $1\left(T_{c}, \Delta_{k}\right)=(1,4)$. Evolution of the tumor. Comparison between the proposed strategy and the uniform maximal injection strategy. Note that the two strategies coincide on the first cycle in order to avoid transient on the observer state evolution.

Real/estimated $G$ and the corresponding error

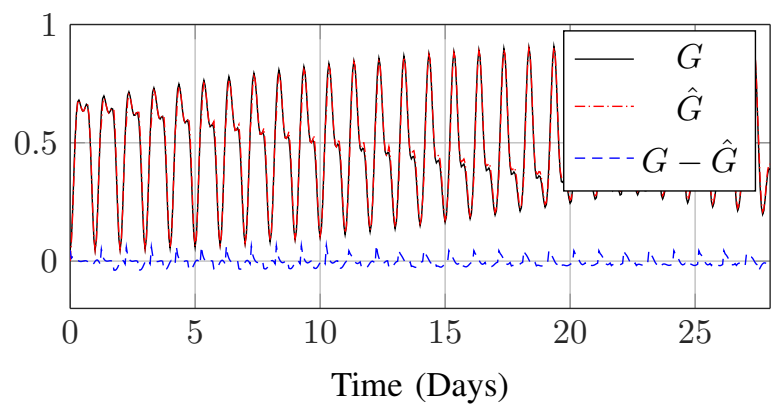

Fig. 2. Case $1\left(T_{c}, \Delta_{k}\right)=(1,4)$. Evolution of the phase-dependent gain $G$ together with its estimated value $\hat{G}$ and the corresponding estimation error. Note that the evolution is not rigorously periodic because of the discrepancies $\delta_{i j}$ (34) affecting the individual pulsations. The persistence of the estimation error is due to the approximation according to which $\dot{\psi}=0$ while $\psi$ is dynamically varying.

constant profile, the observer-based strategy tends to inject the drug during phases where the gain is high in order to enhance the efficiency of the treatment.

- Figures 5 and 6 shows the evolution of the tumor and the corresponding injection profiles in the case where the cycles duration is slightly different $\left(T_{c}=1.1\right.$ instead of $T_{c}=1$ for the first scenario) and the available drug is drastically lower $\left(\Delta_{k}=1\right.$ instead of $\left.\Delta_{k}=4\right)$. Note that in this case, the uniform maximal injection is almost unsuccessful as the tumor size remains almost constant while the observer-based optimal strategy enables a better use of the small available drug in order to enhance the decrease (although slow) of the tumor.

\section{CONCLUSION AND FUTURE WORK}

In this paper, an observer-based drug injection protocol is proposed for the case where the drug is cell-cycle specific, namely, when its efficiency on a given cell depends on the current phase of the cell's cycle. In its current form, the success of the strategy depends on the knowledge of the cycle period and the terms involved in the dynamic 
Real/estimated $G$ and the corresponding error

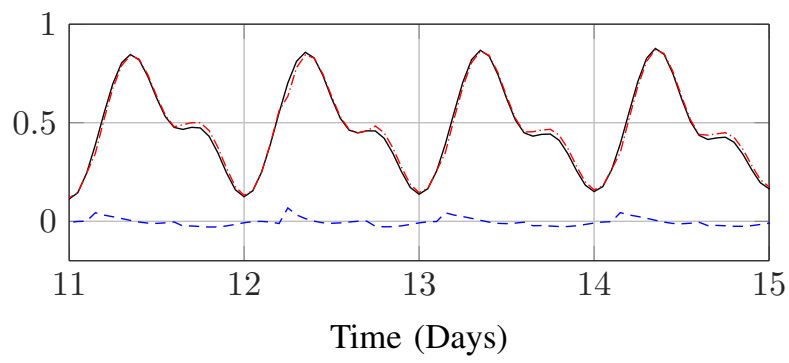

Fig. 3. Case $1\left(T_{c}, \Delta_{k}\right)=(1,4)$. Zoom on Figure 2 showing four successive cycles.

Evolution of drug injection intensity $u$

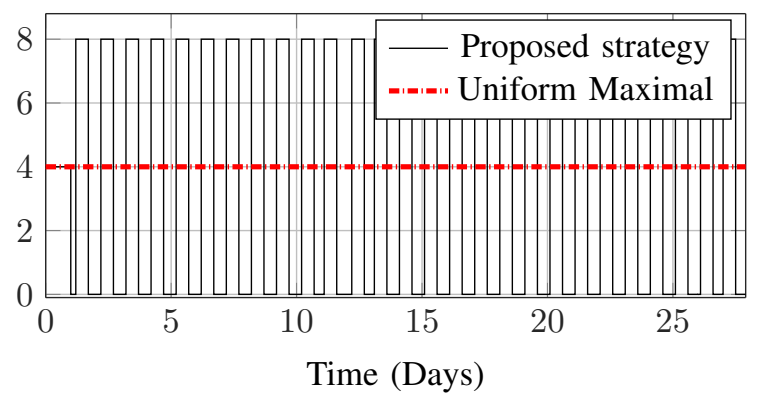

Fig. 4. Case $1\left(T_{c}, \Delta_{k}\right)=(1,4)$. Evolution of the injection intensity $u$ for the two strategies.

of the tumor as this knowledge is used to reconstruct the time-evolution of the gain affecting the drug's concentration in the tumor dynamical equation.

Although the current implementation accommodates for slight errors on the vector of pulsations, further investigation should more seriously evaluate the robustness of the proposed scheme against model mismatch affecting the remaining terms. Moreover, the extension to combined therapy in which one of the drug at least is cell-cycle specific is to be investigated.

\section{REFERENCES}

[1] M. Alamir. On probabilistic certification of combined cancer therapies using strongly uncertain models. Journal of Theoretical Biology, 384:59 - 69, 2015.

[2] B. Alberts, A. Johnson, and J. Lewis et al. Molecular Biology of the Cell. New York: Garland, 2002.

[3] L. G. DePillis and A. E. Radunskaya. A mathematical tumor model with immune resistance and drug therapy: An optimal control approach. Journal of Theoretical Medicine, 3:79-100, 2005.

[4] Yusri A Elsayed and Edward A Sausville. Selected novel anticancer treatments targeting cell signaling proteins. The oncologist, 6(6):517537, 2001.

[5] S. N. Gardner. Cell cycle phase-specific chemotherapy: Computation methods for guiding treatment. Cell cycle, 6(1):369-374, 2002.

[6] K. Kassara and A. Moustafid. Angiogenesis inhibition and tumorimmune interactions with chemotherapy by a control set-valued method. Mathematical Biosciences, 231(2):135 - 143, 2011.

[7] L Marcu, E Bezak, I Olver, and T Van Doorn. Tumour resistance to cisplatin: a modelling approach. Physics in medicine and biology, 50(1):93, 2004.

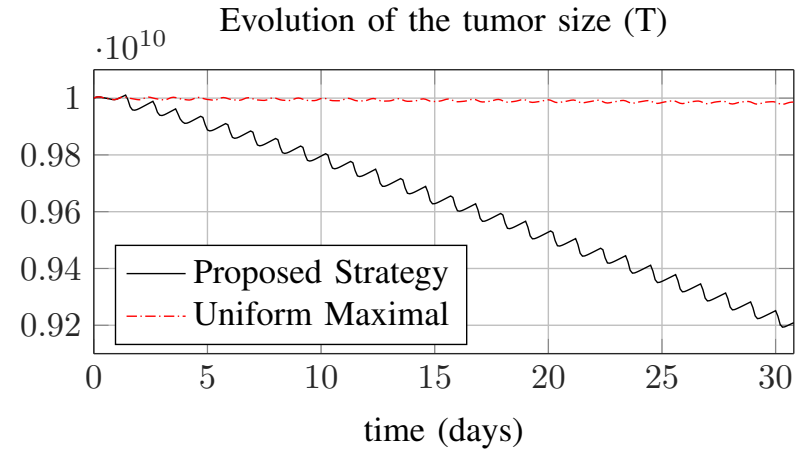

Fig. 5. Case $1\left(T_{c}, \Delta_{k}\right)=(1.1,1)$. Evolution of the tumor. Comparison between the proposed strategy and the uniform maximal injection strategy. Note that the small available drug quantity emphasizes the need of the proposed strategy.

\section{Evolution of drug injection intensity $u$}

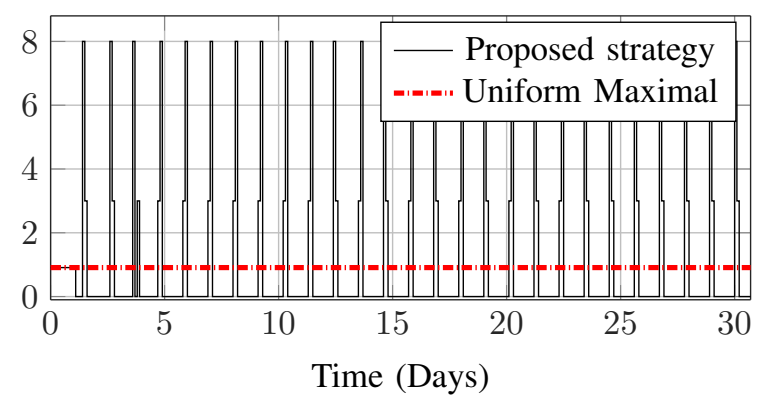

Fig. 6. Case $1\left(T_{c}, \Delta_{k}\right)=(1.1,1)$. Evolution of the injection intensity $u$ for the two strategies.

[8] A. Matveev and A. V. Savkin. Application of optimal control theory to analysis of cancer chemotherapy regimens. Systems and Control Letters, 46:311-321, 2002.

[9] John Carl Panetta and K Renee Fister. Optimal control applied to cell-cycle-specific cancer chemotherapy. SIAM Journal on Applied Mathematics, 60(3):1059-1072, 2000.

[10] Namrata Rastogi and Durga Prasad Mishra. Therapeutic targeting of cancer cell cycle using proteasome inhibitors. Cell division, 7(1):1, 2012.

[11] A. J. Sawyer, J. M. Piepmeier, and W. M. Saltzman. New methods for direct delivery of chemotherapy for treating brain tumor. Yale $J$. Biol. Med., 79(3-4):141-152, 2006.

[12] Manish A Shah and Gary K Schwartz. Cell cycle-mediated drug resistance an emerging concept in cancer therapy. Clinical Cancer Research, 7(8):2168-2181, 2001.

[13] Jeffrey A Silverman and Steven R Deitcher. Marqibo®(vincristine sulfate liposome injection) improves the pharmacokinetics and pharmacodynamics of vincristine. Cancer chemotherapy and pharmacology, 71(3):555-564, 2013.

[14] A Swierniak, A Polanski, and M Kimmel. Optimal control problems arising in cell-cycle-specific cancer chemotherapy. Cell proliferation, 29(3):117-139, 1996.

[15] John J Tyson and Bela Novak. Temporal organization of the cell cycle. Current Biology, 18(17):R759-R768, 2008.

[16] Katrien Vermeulen, Dirk R Van Bockstaele, and Zwi N Berneman. The cell cycle: a review of regulation, deregulation and therapeutic targets in cancer. Cell proliferation, 36(3):131-149, 2003. 